\title{
EuroBIC Medal 2014
}

\author{
Kay Severin
}

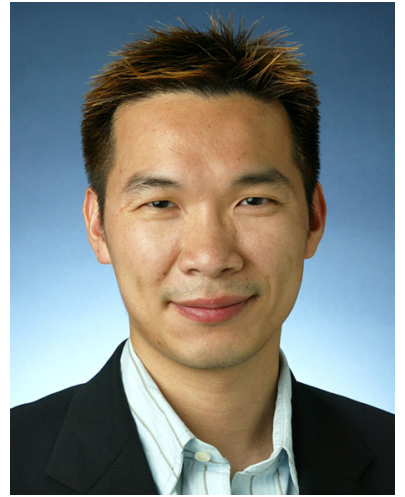

Xile $\mathrm{Hu}$

\section{Award lecture PL 6}

Born in 1978 in Fujian, China, Xile Hu received a Bachelor's degree from Peking University in 2000 and a Ph.D. degree from the University of California, San Diego in 2004. Following a postdoctoral study in the California Institute of Technology from 2005 to 2007, he was appointed tenure-track assistant professor in inorganic chemistry at the Ecole Polytechnique Fédérale de Lausanne (EPFL) in Switzerland. He was promoted to associate professor with tenure at EPFL in 2013. Among his many research interests is the biomimetic and bio-inspired chemistry of redox-active metalloenzymes. During the last several years, he has developed an exciting and highly successful research program in the bio-mimetic chemistry of [Fe]-hydrogenase that forms the basis for this year's EuroBIC Medal.

Hydrogenases are enzymes that catalyze the production and utilization of hydrogen in the biosphere. Given the essential role of hydrogen in energy technology (fuel cell) and chemical synthesis (hydrogenation), the study of hydrogenases is of significant contemporary interest. One exciting recent story in bioinorganic chemistry is the discovery and structural elucidation of [Fe]-hydrogenase. R. Thauer, S. Shima, and co-workers show that [Fe]-hydrogenase is a mononuclear Fe-containing enzyme with a unique pyridinylacyl ligand. The proposed active site is unprecedented in both biology and synthetic chemistry that it warrants confirmation by model complexes. This task is largely accomplished by Xile Hu's research in the area.
In 2010, the Hu group synthesized the first Fe acyl complexes that reproduced the donor set of the active site of [Fe]-hydrogenase, namely, one acyl carbon, one sulfur, two cis- $\mathrm{CO}$, and one pyridinyl nitrogen. This work, published in J. Am. Chem. Soc. 2010, 132, 928-929, demonstrated that an iron complex with the donor set proposed for the active site of [Fe]-hydrogenase could exist outside a protein environment. The work was a strong encouragement for the biomimetic community to explore the model chemistry of [Fe]-hydrogenase.

Later in 2010, the Hu group used model chemistry to confirm another major uncertainty in the proposed active site of $[\mathrm{Fe}]-$ hydrogenase, the pyridinyl acyl ligand. They elegantly applied organometallic synthetic chemistry to install an acylmethylpyridinyl ligand on Fe. Subsequent transformation led to the first model complex to mimic this unique prosthetic group (Angew. Chem. Int. Ed. 2010, 49, 7512-7515). This achievement was not only a synthetic triumph, it also cleared the remaining doubts in the proposed structure of [Fe]-hydrogenase.

In 2011, the Hu group reported an unexpected finding: they found that a model complex reproducing the structural features of $[\mathrm{Fe}]-$ hydrogenase existed as a five-coordinate species (Angew. Chem. Int. $E d$. 2011, 50, 5670-5672). This was a highly relevant finding because X-ray crystallography study of [Fe]-hydrogenase was not able to reveal whether the active site was five or six-coordinate. Furthermore, the catalytically active state of iron has to be five-coordinate to enable the chemistry. This work set the stage for reactivity study of model complexes.

In 2012, using the five-coordinate model mentioned above, the $\mathrm{Hu}$ group demonstrated that the thiolate ligand could be reversibly protonated (Angew. Chem. Int. Ed. 2012, 51, 1919-1921). This reactivity provided insight into the proton transport process during $\mathrm{H}_{2}$ activation in [Fe]-hydrogenase. A proton is yielded during $\mathrm{H}_{2}$ activation; the proton acceptor is however not clear. Xile Hu's chemistry showed that the thiolate ligand in the active site of [Fe]-hydrogenase was a viable proton acceptor.

The attention of the Hu group then turned to $\mathrm{H}_{2}$ activation using model complexes. They found that the model complexes had similar spectroscopic properties to the [Fe]-hydrogenase, and they also exhibited similar ligand binding properties towards $\mathrm{CO}, \mathrm{CN}^{-}$, and isocyanide. However, the models do not react with $\mathrm{H}_{2}$ while the enzyme does. His group applied DFT calculations to probe the origin of this difference. They found that the binding of $\mathrm{H}_{2}$ was the main problem in synthetic compounds as it is unfavorable by about $10 \mathrm{kcal} /$ mol (Eur. J. Inorg. Chem. 2013, 3993-3999). Once $\mathrm{H}_{2}$ is bound, splitting of $\mathrm{H}_{2}$ is facile. Xile $\mathrm{Hu}$ proposed that the enzyme was 
efficient because it used the protein scaffold and the substrate to achieve favorable $\mathrm{H}_{2}$ binding.

This leads to the latest and a very impressive result of his research in the area. In a collaboration with Dr. S. Shima, they successfully incorporated some of his model complexes into the apoenzyme of $[\mathrm{Fe}]$-hydrogenase. Spectroscopic data indicate that the artificial enzymes have a similar electronic property to the native enzyme; reactivity essays confirm the hydrogenase activity of the artificial enzymes. These results provide unprecedented, molecular-level insights into the mechanism of [Fe]-hydrogenase activity. The work is not only a convincing validation of Xile Hu's model chemistry, but also a great example of a new trend in biomimetic chemistry-the marriage between coordination chemistry and enzymology.

In summary, Xile Hu's work in the biomimetic chemistry of [Fe]hydrogenase is a truly outstanding example of bioinorganic chemistry in Europe and is now duly recognized with this year's EuroBIC Medal.

Kay Severin, EPFL Ecole Polytechnique Fédérale de Lausanne (EPFL), Switzerland 